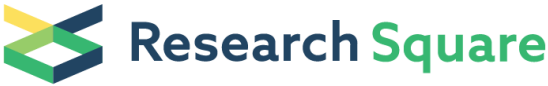 \\ Preprints are preliminary reports that have not undergone peer review. They should not be considered conclusive, used to inform clinical practice, or referenced by the media as validated information.
}

\section{ZNF224 is a Mediator of TGF- $\beta$ Pro-oncogenic Function in Melanoma}

\section{Elena Cesaro}

University of Naples Federico II Department of Molecular Medicine and Medical Biotechnology:

Universita degli Studi di Napoli Federico II Dipartimento di Medicina Molecolare e Biotecnologie Mediche

Arianna Pastore

University of Naples Federico II Department of Molecular Medicine and Medical Biotechnology:

Universita degli Studi di Napoli Federico II Dipartimento di Medicina Molecolare e Biotecnologie Mediche

\section{Alessia Polverino}

University of Naples Federico II Department of Molecular Medicine and Medical Biotechnology:

Universita degli Studi di Napoli Federico II Dipartimento di Medicina Molecolare e Biotecnologie Mediche

\section{Lorenzo Manna}

University of Naples Federico II Department of Molecular Medicine and Medical Biotechnology:

Universita degli Studi di Napoli Federico II Dipartimento di Medicina Molecolare e Biotecnologie Mediche

\section{Giuseppina Divisato}

University of Naples Federico II Department of Molecular Medicine and Medical Biotechnology:

Universita degli Studi di Napoli Federico II Dipartimento di Medicina Molecolare e Biotecnologie Mediche

\section{Cristina Quintavalle}

Institute for Endocrinology and Oncology: Istituto per l'endocrinologia e l'oncologia Gaetano Salvatore

\section{Maddalena Di Sanzo}

Magna Græcia University of Catanzaro Department of Experimental and Clinical Medicine: Universita degli Studi Magna Graecia di Catanzaro Dipartimento di Medicina Sperimentale e Clinica

\section{Maria Concetta Faniello}

Magna Græcia University of Catanzaro Department of Experimental and Clinical Medicine Gaetano Salvatore: Universita degli Studi Magna Graecia di Catanzaro Dipartimento di Medicina Sperimentale e Clinica

\section{Michela Grosso}

University of Naples Federico II Department of Molecular Medicine and Medical Biotechnology:

Universita degli Studi di Napoli Federico II Dipartimento di Medicina Molecolare e Biotecnologie Mediche

\section{Paola Costanzo ( $\square$ paola.costanzo@unina.it)}

Universita degli Studi di Napoli Federico II

\section{Research Article}

Keywords: ZNF224, TGF- $\beta$, melanoma, EMT 
Posted Date: March 6th, 2021

DOI: https://doi.org/10.21203/rs.3.rs-278700/v1

License: (c) (i) This work is licensed under a Creative Commons Attribution 4.0 International License. Read Full License 


\section{Abstract}

Background: The zinc finger protein ZNF224 plays a dual role in human cancers, operating as both tumour suppressor and oncogenic factor depending on the cellular context and molecular partners.

In this research, we investigated the role played by ZNF224 in the TGF- $\beta$ signalling in malignant melanoma.

Methods: Real-time qPCR, western blot, and chromatin immunoprecipitation assays were performed to examine the molecular mechanisms of ZNF224 in TGF- $\beta$ signalling in melanoma. ZNF224-induced cell anchorage, independent growth, migration, and invasion were assessed by the colony formation, wound healing, and transwell assays.

Results: Our findings showed that ZNF224, whose expression increased in melanoma cell lines after TGFb stimulation, potentiated the activation induced by TGF- $\beta$ on its target genes involved in epithelialmesenchymal transition (EMT). Accordingly, overexpression of ZNF224 improved the tumourigenic properties of melanoma cells, promoting cell proliferation and invasiveness, while ZNF224 knockdown had the opposite effect. Moreover, ZNF224 promoted the transcriptional activation of TGF- $\beta$ itself and its type 1 and 2 receptors (T $\beta R 1$ and T $\beta R 2$ ), thus highlighting a possible mechanism by which ZNF224 could enhance the endogenous TGF $\beta /$ Smad signalling.

Conclusions: Our results provide evidence for the involvement of ZNF224 in TGF- $\beta$ signalling as a mediator of TGF- $\beta$ pro-oncogenic function and unveil a positive regulatory loop between TGF- $\beta$ and ZNF224 to promote EMT, consequently increasing the tumour metastatic potential.

\section{Background}

ZNF224 is a ubiquitous transcription factor with a growing number of known activities, molecular partners, and transcriptional targets. It was initially identified as a transcriptional repressor of genes involved in metabolic pathways $(1,2)$. Later, a dual role for ZNF224 as a tumour suppressor or oncogene in human cancer was highlighted.

In chronic myelogenous leukaemia (CML), ZNF224 plays a key pro-apoptotic and antiproliferative role, acting as a transcriptional cofactor of Wilms' tumour protein 1 , WT $1(3,4)$ and operating as a transcriptional repressor of the oncogene c-myc (5) and receptor tyrosine kinase Axl (6).

Conversely, an important oncogenic role for ZNF224 was reported in different cancer types. In these cases, ZNF224 contributes to the impaired growth and apoptosis resistance of cancer cells, operating through the direct or indirect transcriptional modulation of genes involved in proliferation and cell survival $(7,8,9)$. These findings show the dual functions of ZNF224 in cancer, depending on specific sets of interactors and different cellular contexts. 
Melanoma is a highly invasive and metastatic cancer that begins in melanocytes and accounts for $80 \%$ of deaths arising from skin cancer (10). The pleiotropic cytokine TGF- $\beta$ plays a relevant role in melanoma progression. In normal melanocytes, TGF- $\beta$ exerts a potent antiproliferative activity, while in advanced melanoma, it acts as a tumour promoter $(11,12)$, favouring cancer cell proliferation and escape from immune surveillance and later invasion and metastasis, thus stimulating tumour cells to undergo the $\operatorname{EMT}(13,14)$.

Melanoma cells produce large amounts of TGF- $\beta$ isoforms, whose expression correlates with the tumour stage and metastatic progression in patients $(12,15)$. The TGF- $\beta$ pathway is constitutively activated in melanoma cells in response to autocrine TGF- $\beta$ secretion (16). This activation is crucial in promoting melanoma progression through the induction of EMT (11).

In this study, we investigated the role of ZNF224 as a mediator of TGF- $\beta$ pro-oncogenic functions in melanoma. A deeper knowledge of the contribution of ZNF224 as a modulator of TGF- $\beta$ signalling may lead to a better understanding of the molecular mechanisms underlying tumour promoting functions of TGF- $\beta$ in melanoma and to novel treatment options.

\section{Materials And Methods}

\section{Cell cultures and treatments}

A375 and A2058 melanoma cell lines were provided by the Cell Culture Facility of CEINGE (Naples, Italy). The melanoma cell line SAN was established from a patient's biopsy (17).Cells were grown in Dulbecco's Modified Eagle's Medium (DMEM) (Corning, New York, USA) supplemented with 10 or $15 \%$ Fetal Bovine Serum (FBS) (Corning) at $37^{\circ} \mathrm{C}$ in $5 \% \mathrm{CO}_{2}$. For TGF- $\beta$ treatments, $\mathrm{A} 375$ cells were plated at a density of 1.5 $\times 10^{5}$ cells/well in a 12-well plate. The next day, cells were treated with TGF- $\beta 1(10 \mathrm{ng} / \mathrm{mL})($ SigmaAldrich, St. Louis, Missouri, USA).

\section{Transient transfection}

For ZNF224 overexpression, A375 and A2058 cells were transfected with the p3X-Flag ZNF224 expression plasmid or p3X-Flag empty vector as a control. Transient transfection experiments were performed using Metafectene (Biontex, Munchen, Germany) according to the manufacturer's instructions.

For ZNF224 knockdown, A375 cells were transfected with short hairpin RNA (shRNA) plasmids SH2351C3 or SH2352E7 and with the short hairpin Green Fluorescent Protein (shGFP) RNA plasmid as a control (Open Biosystems, AL, USA). Alternatively, cells were transfected with Dharmacon ${ }^{\mathrm{TM}}$ ONTARGETplus HumanZNF224 short interfering RNA (siRNA) -SMARTpool or a non-targeting pool as a control at $50 \mathrm{nM}$ final concentrations for $96 \mathrm{hr}$ using Lipofectamine 2000 (Thermo Fisher Scientific, Waltham, MA, USA)

\section{Cell lysates and western blot assays}


Total protein extracts were obtained by cell lysis in Radio-ImmunoPrecipitation Assay (RIPA) Pierce buffer (Thermo Fisher Scientific, Waltham, MA, USA) or as described previously (18). Total protein extracts were resolved by Sodium Dodecyl Sulphate - PolyAcrylamide Gel Electrophoresis (SDS-PAGE) and then transferred to a nitrocellulose membrane with a RTA Transfer Kit (Bio-Rad) and Trans-Blot turbo (Bio-Rad), according to the manufacturer's instructions. The membranes were incubated with the following antibodies: anti-ZNF224 (rabbit polyclonal, T3) (4) diluted 1:300 in Super-Block Blocking Buffer (Thermo Fisher Scientific, Waltham, MA, USA), anti-p-Smad2, anti-Smad2/3, anti-Slug, anti-Snail, anti-Vimentin, anti- $\beta$-Catenin, and anti-N-Cadherin (Cell Signalling Technology, Danvers, MA, USA) anti- $\beta$-Tubulin (Millipore, Burlington, MA, USA), anti-a-actin (Sigma-Aldrich), anti-Flag (Sigma-Aldrich), anti-T $\beta R$ (Abcam, Cambridge, UK) diluted 1:1000 in 3\% PBS-milk, anti-TGF- $\beta 1$ (V) and anti-Smad2 (Santa Cruz Biotechnology, Inc., Dallas, Texas, USA) diluted 1:300 in 3\% PBS-milk. The secondary antibodies were goat-anti-mouse IgG $(\mathrm{H}+\mathrm{L})$-HRP or goat-anti-rabbit IgG $(\mathrm{H}+\mathrm{L})$-HRP conjugated (Bio-Rad, Bio-Rad, Hercules, California, USA) antibodies (1:5000) in 3\% PBS-milk. Signals were detected with ImmunoCruz Western Blotting Luminol Reagent (Santa Cruz Biotechnology) and Clarity Western Blotting Luminol Reagent (Bio-Rad) by enhanced chemiluminescence (ECL). The band intensities were quantified by densitometry using ImageJ software.

\section{RNA extraction, reverse transcription, and real-time q-PCR}

Total RNA was extracted using the Quick-RNA MiniPrep (ZymoSearch, Irvine, CA, U.S.A.), according to the manufacturer's protocol. Reverse-transcription and quantitative real-time PCR were performed as previously described $(7,19)$. The specific primers used were: N-cadherin (Forward: 5'-

TCCAGACCCCAATTCAATTAATATTAC-3'; Reverse: 5'-AAAATCACCATTAAGCCGAGTGA-3'); $\beta$-catenin (Forward: 5'-TGGATGGGCTGCCTCCAGGTGAC-3': Reverse: 5'-ACCAGCCCACCCCTCGAGCCC-3'); Ecadherin (Forward: 5'-GCCTCCTGAAAAGAGAGTGGAAG-3'; Reverse: 5'-TGGCAGTGTCTCTCCAAATCCG-3'); Vimentin (Forward 5'-TACAGGAAGCTGGAAGG-3' Reverse 5'-ACCAGAGGGAGTGAATCCAG-3'); TGFßR1 (Forward: 5'-TCCTGGGATTTATAGCAGCAGAC-3'; Reverse: 5'-CGTGGACAGAGCAAGTTTTATCA-3'); TGFßR2 (Forward: 5'- TCCTTCAAGCAGACCGATGT-3'; Reverse: 5'-GAACCAAATGGAGGCTCATAATC-3)'; HPRT; and ZNF224 (7). Snail, slug, and TGF- $\beta 1$ were validated primers from QuantiTect (Qiagen, Valencia, CA, USA).

The relative quantification in gene expression was determined using the $\Delta \Delta \mathrm{CT}$ method.

\section{Immunofluorescence assay}

A375 cells were plated on coverslips and transfected with the p3X-Flag ZNF224 expression plasmid or p3X-Flag empty vector. Forty-eight hours post-transfection, the cells were washed with 1X Phosphate Buffered Saline (PBS) and fixed with $4 \%$ paraformaldehyde (PFA) for $10 \mathrm{~min}$ at room temperature. The cells were permeabilised with $0.1 \%$ Triton X-100 (AppliChem, Ottoweg, Germany) for 5 min and incubated with the blocking solution for $30 \mathrm{~min}$ at room temperature. For antigen detection, the cells were incubated with the primary antibody N-Cadherin (D4R1H) XP® Rabbit mAb \#13116 (1:100, Cell Signalling Technology) overnight at $4^{\circ} \mathrm{C}$. The following day, the coverslips were washed with $1 \mathrm{X}$ PBS and incubated with the secondary antibody IgG $(\mathrm{H}+\mathrm{L})$ Highly Cross-Adsorbed Donkey anti-Rabbit, Alexa Fluor ${ }^{\mathrm{rm}} 488$ 
(Invitrogen, USA) for $1 \mathrm{~h}$ at room temperature. The nuclei were stained with DAPI, Dihydrochloride (Calbiochem, San Diego, USA) for $5 \mathrm{~min}$ at room temperature. A Leica Thunder Imaging System (Leica Microsystems Wetzlar, Germany) equipped with a LEICA DFC9000 GTC camera, lumencor fluorescence LED light source, and $63 \mathrm{X}$ oil immersion objective was used to acquire Z-slice images. Small volume computational clearing (SVCC) was used to remove the background signal derived from out-of-focus blur.

\section{Chromatin immunoprecipitation assay}

Chromatin immunoprecipitation was performed as previously described $(7,20)$. Briefly, forty-eight h posttransfection with FlagZNF224 plasmid, A375 cells were cross-linked with $\mathrm{HCHO}(1 \%)$ for 10 min at room temperature, lysed, and fixed chromatin was sheared using an ultrasonic liquid processor. Chromatin was immunoprecipitated overnight on the wheel at $4^{\circ}$ with $1 \mu \mathrm{g}$ anti-Flag antibody (Sigma) or $1 \mu \mathrm{g} \lg \mathrm{G}$ (Sigma). On the following day, the immunocomplexes were recovered by protein $A / G$ plus Agarose (Santa Cruz). The isolated complexes were washed twice in RIPA buffer (0.1\% SDS, 1 mM EDTA, 0.5 mM EGTA, $1 \%$ Triton X-100, 0.1\% Na-Deoxycholate, $10 \mathrm{mM}$ Tris- $\mathrm{HCl}(\mathrm{pH} 8)$, and $140 \mathrm{mM} \mathrm{NaCl}$ ), four times in $10 \mathrm{mM}$ Hepes (pH 8), $0.1 \%$ Igepal, $5 \mathrm{mM}$ EDTA, and $250 \mathrm{mM} \mathrm{NaCl}$ solution, and once in $10 \mathrm{mM}$ Tris (pH 8) and 1 mM EDTA. Crosslinking was reversed at $65^{\circ} \mathrm{C}$ overnight in $10 \mathrm{mM}$ Tris- $\mathrm{HCl}(\mathrm{pH} 8), 1 \mathrm{mM}$ EDTA, and $1 \%$ SDS. Subsequently, DNA was recovered by phenol/chloroform extraction and ethanol precipitation. The analysis of immunoprecipitated DNA and input controls was performed in triplicate by quantitative realtime PCR using a Master Mix SYBRGreen (Bio-Rad). The Ct values were calculated by using appropriate Biorad software. Relative enrichment was calculated as fold enrichment, obtained by subtracting the $\mathrm{Ct}$ value for the IgG antibody background from the $\mathrm{Ct}$ value for the antibody of interest (Flag): $2^{\wedge}$-(Ct IP-Ct $\mathrm{lgG}$ ). The negative sample was given a value of 1. Primer sequences were as follows: TGF $\beta 1$ (Forward: GAACTGTGTTCTGAGGACATGG; Reverse: CCTCTCTGTGTTATCCTCCTCC); TGFßR1 (Forward: GCTTTGCTAAAAGCTGGAGGAGGAT; Reverse: TAAATGTCTGGCTCTGCCTTTG); TGFßR2 (Forward: AAGGGATAGCTCTGTGTGTGTG; Reverse: AAGAGAGACATCATCCTGAGCC); and unrelated region (UNR) (Forward: CTGACAAGGTGATGGGCTTATG; Reverse: AAGGATTCGGTGATGGCTCTA)

\section{Colony formation assay}

A375 and A2058 cells overexpressing or silenced for ZN224 and their respective control cells were detached $24 \mathrm{~h}$ after transfection and seeded at a density of $5 \times 10^{2}$ in a 6-well plate in triplicate and incubated for 15 days. The culture medium was replaced every 2 days. After fixing with $25 \%$ methanol and staining with $0.1 \%$ Crystal Violet, the colonies were counted. The average colony count for the three dishes was used to calculate the plating efficiency (plating efficiency = number of colonies counted/number of cells plated). After elution of Crystal Violet with $1 \%$ SDS, absorbance at a $570 \mathrm{~nm}$ wavelength was measured using a Microplate Reader -BioTek Synergy H1 (BioTek U.S., Winooski, VT, USA)

\section{Migration and invasion assays}

The migration of A375 cells was evaluated using Transwell Supports for 24-well plates with an $8 \mu \mathrm{m}$ pore membrane size (Falcon, Corning Inc.). For the invasion assay, the upper side of the Transwell Supports 
(Corning) was precoated with $100 \mu \mathrm{l}$ of Matrigel Basement Membrane Matrix (Corning) diluted 1:5 in DMEM free medium and allowed to dry out at $37^{\circ} \mathrm{C}$ for at least $1 \mathrm{~h}$. In the lower panel, $600 \mu \mathrm{l}$ of DMEM supplemented with $15 \%$ FBS was added. A375 cells overexpressing or silenced for ZN224 were seeded on the upper side of the membrane at a density of $2 \times 10^{4}$ cells/100 $\mu$ l of DMEM supplemented with FBS $1 \%$. After $20 \mathrm{~h}$ of incubation at $37^{\circ} \mathrm{C}$, cells on the upper surface of the membrane were removed using a cotton wool swab and migrated or invasive cells on the lower side of the membrane were fixed with $25 \%$ methanol and stained with $0.1 \%$ Crystal Violet. The images of stained cells were captured under a light microscope (Leica DFC365 FX, Leica Microsystem, Wetzlar, Germany) at a magnification of $\times 5-\times 10$ in 5 random fields in each well. The percentage of migratory and invasive cells was evaluated by eluting fixed cells with $1 \%$ SDS and reading the absorbance at $\lambda 570 \mathrm{~nm}$.

\section{Soft-agar assay}

A375 and A2058 cells transiently transfected with 3xFlag-ZNF224 or the 3xFlag empty vector were used to evaluate anchorage-independent growth. Dishes $(60 \mathrm{~mm})$ were precoated with a solution containing DMEM 2× (Sigma, St Louis, MO, USA), Tryptose Phosphate Broth (TPB) Buffer (Difco, BD, Franklin Lakes, NJ, USA) and 1.25\% Noble Agar (Difco, BD, Franklin Lakes, NJ, USA) and left to dry for $10 \mathrm{~min}$. Next, $10^{4}$ cells were resuspended in $2 \mathrm{ml}$ AGAR DMEM and plated on top of the dried Noble Agar layer in the $60 \mathrm{~mm}$ dishes. Cells were grown for 2 weeks in the incubator at $37^{\circ} \mathrm{C}$ in $5 \% \mathrm{CO}$, and fresh medium was added once a week. Cell clumps were observed, and their pictures were captured under a light microscope (Leica DFC365 FX, Leica Microsystem, Germany) at a magnification of $\times 5$ in 5 random fields in each well. Cell colonies were counted using ImageJ software (Version 1.49).

\section{Wound healing assay}

A2058 cells overexpressing ZNF224 and control cells (Flag) were seeded in $60 \mathrm{~mm}$ dishes at a density of $4 \times 10^{5}$. After $24 \mathrm{~h}$, a yellow pipette tip was used to make a scratch. Cells were rinsed three times with $1 \mathrm{X}$ PBS and once with growth medium to remove the detached cells. Then, $3 \mathrm{ml}$ of fresh DMEM were added. Scratch closure was monitored, and images were captured at 0,24 , and $48 \mathrm{~h}$ using a light microscope (Leica DFC365 FX, Leica Microsystem, Germany). Wound closure was measured by calculating the density of the pixels in the area where the cut was made and expressed as a percentage of wound closure in the area. The percentage of wound closure was calculated by Image $J$ software (Version 1.49)

\section{Statistical analyses}

Data were presented as the mean \pm standard error of the mean (SEM) from three or more independent experiments unless indicated otherwise. Statistical analysis was performed with Prism $7^{\mathrm{TM}}$ (GraphPad Software Inc., La Jolla, CA, USA). $P \leq 0.05$ was considered a significant difference $\left({ }^{\star} P \leq 0.05 ;{ }^{\star \star} P \leq 0.01\right)$.

\section{Results}




\section{ZNF224 expression was induced by TGF- $\beta$ and affected the TGF- $\beta$ signalling pathway}

The analysis of ZNF224 protein levels in various melanoma cell lines and non-cancerous human fibroblasts, performed by western blot, showed that malignant cells exhibited higher ZNF224 expression than normal cells (Fig. 1A). Furthermore, ZNF224 expression was induced by TGF- $\beta$ in a time-dependent manner in A375 cells. TGF- $\beta$-induced Smad2 phosphorylation was analysed to verify the activation of signalling pathways downstream of TGF- $\beta$ (Fig. 1B).

To evaluate if ZNF224 expression could affect the TGF- $\beta$ /Smad pathway in melanoma cells, we examined the phosphorylation status of Smad2 protein in A375 cells transfected with a p3xFlagZNF224 expression vector. In A375 transfected with an empty control vector (Flag), the phosphorylation of Smad2 was detected $1 \mathrm{~h}$ after TGF- $\beta$ stimulation and gradually decreased as expected, while the overexpression of ZNF224 was accompanied by prolonged Smad2 phosphorylation (Fig. 1C). This result suggests that high ZNF224 expression may play a role in sustaining TGF- $\beta /$ Smad signalling in A375 cells, thus affecting the expression of TGF- $\beta$ regulated genes.

In support of this hypothesis, we examined mRNA and protein levels of some TGF- $\beta$ target genes in A375 cells overexpressing ZNF224 and found altered expression, thus indicating that ZNF224 mimics the TGF$\beta$ effect (Fig. 2A, B). Specifically, we observed increased expression of the transcription factors Slug and Snail, regulators of TGF- $\beta$-induced EMT, and the mesenchymal markers Vimentin and N-cadherin. Interestingly, ZNF224 overexpression was also accompanied by decreased mRNA expression of the epithelial marker E-cadherin and increased expression of $\beta$-catenin, whose signalling is activated in melanoma progression and promotes growth and survival of melanoma cells $(21,22)$. Immunofluorescence staining confirmed the increased expression of N-cadherin in A375 cells overexpressing ZNF224 (Fig. 2C).

Subsequently, to evaluate the effects of ZNF224 on the TGF- $\beta$ induced expression of its target genes, A375 cells were transfected with Flag-ZNF224 or Flag as a control and then treated with TGF- $\beta$ for different lengths of time. Notably, the expression of N-cadherin, $\beta$-catenin, Slug, and Snail mRNA was induced by TGF- $\beta$ as expected but was significantly increased further in the presence of ZNF224 overexpression (Fig. 3A).

These results highlight a synergistic effect between ZNF224 and TGF- $\beta$ in the regulation of TGF- $\beta$ target genes. We further confirmed the existence of this cooperative effect by Western blot analysis (Fig. 3B)

We also examined the effects of siRNA-mediated knockdown of ZNF224 on the expression of these genes. Silencing of ZNF224 (Fig. 3C) was accompanied by reduced mRNA expression of N-cadherin, $\beta$ catenin, Slug, Snail, and Vimentin, thus indicating that ZNF224 depleted cells fail to activate EMTpromoting factors (Fig. 3D). 
Furthermore, we confirmed the effect of ZNF224 in sustaining TGF- $\beta /$ Smad signalling by evaluating the TGF- $\beta$ induced expression of N-cadherin, $\beta$-catenin, Slug, and Snail in A375 cells silenced for ZNF224. Figure $3 \mathrm{E}$ shows that silencing of ZNF224 before TGF- $\beta$ treatment counteracted the TGF-induced expression of these targets compared with cells transfected with scrambled siRNA, indicating that ZNF224 is required for TGF- $\beta$ dependent regulation of its target genes.

\section{ZNF224 positively modulated the expression of TGF- $\beta$, TGF- $\beta R 1$, and TGF- $\beta R 2$}

TGF- $\beta$ signalling is subjected to fine-tuning by a variety of regulators operating at different levels of the TGF- $\beta$ pathway and both positive and negative feedback loops. Modulation of TGF- $\beta$ production and TGF- $\beta$ receptors activity is a critical step for signalling regulation.

In particular, to amplify its signalling, the TGF- $\beta$ ligand induced a rapid translocation of its receptors to the cell surface (23). Furthermore, TGF- $\beta$ also stimulated its own expression and expression of its receptors (24).

To investigate the molecular mechanism by which ZNF224 takes part in the modulation of TGF- $\beta$ signalling, we measured the mRNA levels of TGF- $\beta$ and type 1 and 2 TGF- $\beta$ receptors in A375 cells overexpressing or silenced for ZNF224. Interestingly, we found increased expression of TGF- $\beta$, TGF- $\beta R 1$, and TGF- $\beta$ R2 because of ZNF224 overexpression (Fig. 4A left panel). Conversely, ZNF224 silencing resulted in reduced expression of these mRNAs (Fig. 4A Right panel). Western blot analysis confirmed the induced expression of TGFBR1 and TGF- $\beta$ following ZNF224 overexpression (Fig. 4B).

Subsequently, by in silico analysis, we found ZNF224 binding motifs (6) in the promoter region of TGF- $\beta$ and in the first intron of TGF- $\beta R 1$ and TGF- $\beta$ R2 genes. A chromatin immunoprecipitation assay (X-ChIP) was performed to verify ZNF224 occupancy on these genomic regions. The crosslinked chromatin of A375 cells transfected with the FlagZNF224 plasmid were immunoprecipitated with a Flag antibody. RTqPCR analysis confirmed the binding of ZNF224 to its consensus sequences on the TGF- $\beta$, TGF $\beta R 1$, and TGF-BR2 genes (Fig. 4C).

These results indicated that ZNF224 sustains endogenous TGF $\beta / S$ mad signalling by upregulating the expression of TGF- $\beta$ R1, TGF- $\beta$ R2, and TGF- $\beta$.

Accordingly, as shown in Fig. 4D, ZNF224 overexpression further increased the expression of TGF- $\beta$ and its receptors compared to the induction observed in the presence of TGF- $\beta$ treatment alone. These results indicate that ZNF224 potentiated TGF- $\beta$ signalling in melanoma cells through the simultaneous activation of the cytokine and its receptors, which are essential for TGF-induced effects (23)

\section{ZNF224 promoted anchorage-independent growth, migration, and invasion of melanoma cells}


To address the functional role of ZNF224 in melanoma cell growth, we first assessed the clonogenic potential of A375 and A2058 cells in condition of overexpression and silencing of ZNF224 (Fig. 5). The number of colonies formed was significantly increased in A375 cells following ZNF224 overexpression compared to the control cells. Conversely, fewer colonies were observed in A375 cells silenced for ZNF224 by shRNA transfection (shC3 and shE7) compared to control cells (shGFP) (Fig. 5A, B). Similar effects were found in A2058 cells (Fig. 5D, E), thus demonstrating that ZNF224 can increase the proliferation rate of melanoma cells.

To evaluate the ability of ZNF224 to promote the anchorage-independent growth of melanoma cells, we performed a soft agar colony formation assay in A375 and A2058 cells overexpressing ZNF224. There was a remarkable increase in the colony-forming ability of both cell lines transfected with Flag ZNF224 compared to cells transfected with the empty vector (Flag) (Fig. 6).

Subsequently, we explored the potential impact of ZNF224 on A375 cells migration and invasion using transwell assays. As shown in Fig. 7, A375 cells transfected with ZNF224 exhibited a significantly increased migratory and invasive potential compared to cells transfected with the empty vector (Flag) (panel A). Conversely, the downregulation of ZNF224 induced by siRNA transfection significantly suppressed cell migration and invasion compared to control cells (panel B). The enhanced migratory ability of cells overexpressing ZNF224 was also demonstrated in A2058 cells through a wound healing assay (Fig. 7C). Overall, these results support the idea that in melanoma cancer cells, ZNF224 promotes the acquisition of anchorage-independent cell growth and migration and invasion abilities, which are critical steps in cancer progression and metastasis.

\section{Discussion}

Melanoma progression and metastasis are complex processes, and the mechanisms that mediate the switch from primary to metastatic melanoma remain poorly understood (25).

TGF- $\beta$ exerts important and pleiotropic effects in melanoma progression. It acts in autocrine and paracrine manners to control and shape tumour growth, invasion, escape from immune surveillance, and metastasis $(11,26)$.

Positive and negative feedback regulatory loops modulate TGF- $\beta$ signalling $(27,28)$. TGF- $\beta$ positively regulates its own expression and induces the upregulation of TGF- $\beta$ receptors $(23,24)$, thus amplifying signalling and Smad-mediated gene responses. TGF- $\beta$ also induces the expression of some transcription factors that may cooperate with Smad in the transcriptional regulation of TGF- $\beta$ target genes $(28,29,30)$.

Increasing evidence indicates a role for the zinc finger protein ZNF224 in carcinogenesis, demonstrating that this transcription factor behaves as a tumour suppressor or oncogene in different types of cancer (31). 
Here, we provided compelling evidence that ZNF224 acts as a positive regulator of TGF- $\beta$ signalling in melanoma, thus contributing to TGF- $\beta$ induced tumour-promoting effects.

Moreover, we found that TGF- $\beta$ induces ZNF224 expression in melanoma cell lines and that ZNF224 overexpression is accompanied by prolonged Smad2 phosphorylation. Furthermore, overexpression and silencing experiments showed that ZNF224 was able to enhance the effects of TGF- $\beta$ on some TGF- $\beta$ inducible genes, such as the EMT-related transcription factors Slug and Snail, which are associated with cell invasion and development of metastases and some epithelial-mesenchymal transition markers.

Although the molecular mechanisms of the transcriptional regulatory functions of ZNF224 within the TGF- $\beta$ pathway were not extensively investigated here, it is conceivable that the DNA-binding activity of ZNF224 may facilitate the recruitment of Smad transcriptional complexes to target promoter sites, favouring high-affinity and selective interactions with cognate DNA. Numerous DNA-binding transcription factors play a crucial role in Smad-controlled target gene selection (28). However, considering that TGF- $\beta$ can also activate intracellular pathways other than Smad-mediated canonical signalling (30), we cannot rule out that ZNF224 could also modulate the transcription of some TGF- $\beta$ target genes in a Smadindependent manner. Moreover, ZNF224 may promote TGF- $\beta$ signalling, activating or repressing other transcriptional regulators of this pathway.

Here, we identified one of the possible mechanisms by which ZNF224 could exert a regulatory role in the TGF- $\beta$ pathway. By chromatin immunoprecipitation experiments and the evaluation of mRNAs expression changes following ZNF224 overexpression or silencing, we demonstrated that TGF- $\beta R 1$, TGF- $\beta R 2$, and TGF- $\beta$ itself are direct target genes of ZNF224 transcriptional activation. The augmented expression of TGF- $\beta$ and its receptors and prolonged Smad2 phosphorylation induced by ZNF224 contributes to the constitutive activation of the pathway, thus resulting in enhanced induction of some TGF- $\beta$-responsive genes, associated with EMT and subsequent malignant progression.

Furthermore, our data highlight the existence of a positive regulatory loop between TGF- $\beta$ and ZNF224 in melanoma. Indeed, we also showed that ZNF224 expression was, in turn, induced by TGF- $\beta$, thus further contributing to the deregulated activation of this pathway.

Altogether, our data show that ZNF224 is required for the proliferation, migration, and invasiveness of melanoma cells. Its overexpression could represent a critical event in the multi-step process that leads to tumour cell invasion and metastasis. Furthermore, ZNF224 could act by promoting the acquisition of a mesenchymal phenotype and the metastatic behaviour of melanoma by modulating different EMT cancer-related proteins.

Great efforts are being made to develop drugs targeting TGF- $\beta$ in melanoma $(32,33,34)$. However, these approaches have proven challenging for rapid application in clinical practice due to the numerous physiological functions in which this signalling pathway is involved $(35,36)$. 
The identification of ZNF224 as one of the modulators of TGF- $\beta$ signalling will provide a deeper knowledge of the molecular events involving this pathway in melanoma progression and invasion. Further experiments will be performed to characterise the molecular network between ZNF224 and TGF- $\beta$, thus contributing to find new strategies for targeting TGF- $\beta$ signalling and, consequently, new molecular therapeutic targets to treat this deadly disease.

\section{Conclusions}

Our findings demonstrate that the zinc finger transcription factor ZNF224 is a positive modulator of the TGF- $\beta$ pathway in melanoma to mediate the pro-oncogenic function of the cytokine.

ZNF224 acts synergistically with TGF-b to regulate genes involved in EMT. Accordingly, overexpression of ZNF224 stimulates the mesenchymal phenotype, increasing the expression of EMT master regulators and mesenchymal markers. Specifically, ZNF224 influences the proliferation, migration, and adhesion capacity of melanoma cells, thus contributing to define a more aggressive tumour phenotype.

Moreover, ZNF224 expression was increased by TGF-b stimulation, and its overexpression induced transcription of TGF- $\beta$ and its receptors, thus sustaining the constitutive activation of TGF- $\beta$ signalling.

Defining the molecular mechanisms underlying the crosstalk between TGF- $\beta$ and ZNF224 might contribute to clarifying the mechanism of resistance to TGF $\beta$-mediated growth inhibition in melanoma, with relevant implications in the development of new therapeutic approaches in melanoma.

\section{Abbreviations}

ZNF224: Zinc Finger Protein 224; TGF- $\beta$ : Transforming Growth Factor beta; qPCR: quantitative Polymerase Chain Reaction; EMT: Epithelial-Mesenchymal Transition; T $\beta R 1$ : Transforming growth factor-beta type 1 Receptor; T $\beta R 2$ : Transforming growth factor-beta type 2 Receptor; SMAD: Small Mother Against Decapentaplegic; CML: Chronic Myelogenous Leukemia; WT1: Wilms' Tumor protein 1; c-myc: cellular myelocytomatosis; AxI: AXL receptor tyrosine kinase; DMEM: Dulbecco's Modified Eagle's Medium; FBS: Fetal Bovine Serum; shRNA: short hairpin RNA; shGFP: short hairpin Green Fluorescent Protein; siRNA: short interfering RNA; RIPA: Radio-immunoprecipitation assay; SDS-PAGE: Sodium Dodecyl Sulphate - Polyacrylamide Gel Electrophoresis; PBS: Phosphate Buffered Saline; HRP: Horseradish Peroxidase; ECL: Enhanced Chemiluminescence; RT- PCR: Reverse-Transcriptase Polymerase Chain Reaction; HPRT: Hypoxanthine guanine Phosphoribosyltansferase; PFA: Paraformaldehyde; SVCC: Small Volume Computational Clearing; X-ChIP: Cross-linked chromatin immunoprecipitation; SDS: Sodium Dodecyl Sulphate; EDTA: Ethylenediaminetetraacetic acid; EGTA: egtazic acid; TPB: Tryptose Phosphate Broth; SEM: Standard error of the mean; ANOVA: Analysis of variance; RT-qPCR: reverse transcription quantitative real-time Polymerase Chain Reaction.

\section{Declarations}




\section{Ethic approval and consent to participate}

Not applicable

\section{Consent for publication}

Not applicable

\section{Availability of data and material}

Not applicable

\section{Competing interests}

The authors declare no conflict of interest

\section{Funding}

Not applicable

\section{Authors'contributions}

EC contributed to the design of the study, planned and analysed experiments, and drafted the manuscript. AP, AP and LM performed the experiments. GD performed the immunofluorescence experiments. CQ, MDS and MCF performed some in vitro functional assays. MG suggested some experiments and revised the final version of the manuscript PC conceived the study, supervised most of the experiments, and wrote the manuscript. All authors read and approved the final manuscript

\section{Acknowledgments}

We thank the Centre of Interdepartmental Services (CIS), "Magna Graecia” University of Catanzaro, Italy for supporting part of this research

\section{References}

1. Cesaro E, De Cegli R, Medugno L, Florio F, Grosso M, Lupo A, et al. The Kruppel-like zinc finger protein ZNF224 recruits the arginine methyltransferase PRMT5 on the transcriptional repressor complex of the aldolase A gene. J Biol Chem. 2009; 284:32321-30. 
2. Iacobazzi V, Infantino V, Convertini P, Vozza A, Agrimi G, Palmieri F. Transcription of the mitochondrial citrate carrier gene: Identification of a silencer and its binding protein ZNF224. Bioch Bioph Res Commun. 2009; 386:186-91.

3. Montano G, Cesaro E, Fattore L, Vidovic K, Palladino C, Crescitelli R, et al. Role of WT1-ZNF224 interaction in the expression of apoptosis-regulating genes. Hum Mol Genet. 2013; 22:1771-82.

4. Montano G, Vidovic K, Palladino C, Cesaro E, Sodaro G, Quintarelli C, et al. WT1-mediated repression of the proapoptotic transcription factor ZNF224 is triggered by the BCR-ABL oncogene. Oncotarget. $2015 ; 6: 28223-37$.

5. Sodaro G, Cesaro E, Montano G, Blasio G, Fiorentino F, Romano S, et al. Role of ZNF224 in c-Myc repression and imatinib responsiveness in chronic myeloid leukemia. Oncotarget. 2018; 9:3417-31.

6. Sodaro G, Blasio G, Fiorentino F, Auberger P, Costanzo P, Cesaro E. ZNF224 is a transcriptional repressor of AXL in chronic myeloid leukemia cells. Biochimie. 2018; 154:127-31.

7. Busiello T, Ciano M, Romano S, Sodaro G, Garofalo O, Bruzzese D, et al. Role of ZNF224 in cell growth and chemoresistance of chronic lymphocitic leukemia. Hum Mol Genet. 2017; 26:344-53.

8. Harada Y, Kanehira M, Fujisawa Y, Takata R, Shuin T, Miki T, et al. Cell permeable peptide DEPDC1ZNF224 interferes with transcriptional repression and oncogenicity in bladder cancer cells. Cancer Res. 2010; 70:5829-39.

9. Cho JG, Park S, Lim CH, Kim HS, Song SY, Roh TY, et al. ZNF224, Kruppel like zinc finger protein, induces cell growth and apoptosis-resistance by downregulation of p21 and p53 via miR-663a. Oncotarget. 2016; 7:31177-90.

10. Shain HA, Bastian BC. From melanocytes to melanomas. Nat Rev Cancer. 2016; 16:345-58.

11. Perrot $C Y$, Javelaud $D$, Mauviel A. Insights into the transforming growth factor- $\beta$ signaling pathway in cutaneous melanoma. Ann Dermatol. 2013; 25:135-44.

12. Javelaud D, Alexaki VI, Mauviel A. Transforming growth factor-beta in cutaneous melanoma. Pigment Cell Melanoma Res. 2008; 21:123-32.

13. Jakowlew SB. Transforming growth factor-beta in cancer and metastasis. Cancer Metastasis Rev. 2006; 25:435-57.

14. Hao Y, Baker D, Dijke PT. TGF- $\beta$-mediated epithelial-mesenchymal transition and cancer metastasis. Int J Mol Sci. 2019; 20:2767.

15. Tang MR, Wang YX, Guo S, Han SY, Li HH, Jin SF. Prognostic significance of in situ and plasma levels of transforming growth factor $\beta 1,-2$ and -3 in cutaneous melanoma. Mol Med Rep. 2015; 11:4508-12.

16. Rodeck U, Nishiyama T, Mauviel A. Independent regulation of growth and SMAD-mediated transcription by transforming growth factor beta in human melanoma cells. Cancer Res. 1999; 59:547-50.

17. Benassi B, Zupi G, Biroccio A. Gamma-glutamylcysteine synthetase mediates the c-Myc-dependent response to antineoplastic agents in melanoma cells. Mol Pharmacol. 2007; 72:1015-23. 
18. Testa G, Russo M, Di Benedetto G, Barbato M, Parisi S, Pirozzi F, et al. Bmi1 inhibitor PTC-209 promotes chemically-induced direct cardiac reprogramming of cardiac fibroblasts into cardiomyocytes. Sci Rep. 2020; 10:1-10.

19. Faniello MC, Di Sanzo M, Quaresima B, Nisticò A, Fregola A, Grosso M, et al. Bilateral cataract in a subject carrying a $C$ to $A$ transition in the $L$ ferritin promoter region. Clin. Biochem. 2009; 42:911-4

20. Passaro F, De Martino I, Zambelli F, Di Benedetto G, Barbato M, D'Erchia AM, et al. YAP contributes to DNA methylation remodeling upon mouse embryonic stem cell differentiation. J Biol Chem. 2020; doi:1074/jbcRA120.015896

21. Widlund HR, Horstmann MA, Price ER, Cui J, Lessnick SL, Wu M, et al. Beta-catenin-induced melanoma growth requires the downstream target Microphthalmia-associated transcription factor. J Cell Biol. 2002; 158:1079-87.

22. Sinnberg T, Menzel M, Ewerth D, Sauer B, Schwarz M, Schaller $M$, et al. $\beta$-Catenin signaling increases during melanoma progression and promotes tumor cell survival and chemoresistance. PLoS One. 2011; 6:e23429.

23. Duan D, Derynck R. Transforming growth factor- $\beta$ (TGF- $\beta$ )-induced up-regulation of TGF- $\beta$ receptors at the cell surface amplifies the TGF- $\beta$ response. J Biol Chem. 2019; 294:8490-504.

24. Menke A, Geerling I, Giehl K, Vogelmann R, Reinshagen M, Adler G. Transforming growth factor-betainduced upregulation of transforming growth factor-beta- receptor expression in pancreatic regeneration. Biochim Biophys Acta. 1999; 1449:178-85.

25. Damsky WE, Theodosakis N, Bosenberg M. Melanoma metastasis: new concept and evolving paradigms. Oncogene. 2014; 33:2413-22.

26. Busse A, Keilholz U. Role of TGF- $\beta$ in melanoma. Curr Pharm Biotechnol. 2011; 12:2165-75.

27. Miyazono K. Positive and negative regulation of TGF-beta signaling. J Cell Sci. 2000; 113:1101-9.

28. Massague J, Wotton D. Transcriptional control by the TGF-b/Smad signaling system. EMBO J. 2000; 19:1745-54.

29. Hill CS. Transcriptional control by the SMADs. Cold Spring Harb Perspect Biol. 2016; 8:a022079.

30. Zhang YE. Non Smad pathways in TGF-b signaling. Cell Res. 2009; 19:128-139.

31. Cesaro E, Sodaro G, Montano G, Grosso M, Lupo A, Costanzo P. The complex role of the ZNF224 transcription factor in cancer. Adv Protein Chem Struct Biol. 2017; 107:191-222.

32. Romano MF. Targeting TGFbeta-mediated processes in cancer. Curr Opin Drug Discov Devel. 2009; 12:253-63.

33. Yingling JM, Blanchard KL, Sawyer JS. Development of TGF-beta signalling inhibitors for cancer therapy. Nat Rev Drug Discov. 2004; 3:1011-22.

34. Neuzillet C, Tijeras-Raballand A, Cohen R, Cros J, Faivre S, Raymond E, et al. Targeting the TGF- $\beta$ pathway for cancer therapy. Pharmacol Ther. 2015; 147:22-31.

35. Connolly EC, Freimuth J, Akhurst RJ. Complexities of TGF- $\beta$ targeted cancer therapy. Int J Biol Sci. 2012; 8:964-78. 
36. Ciardiello D, Elez E, Tabernero J, Seoane J. Clinical development of therapies targeting TGF $\beta$ : current knowledge and future perspectives. Ann Oncol. 2020; 31:1336-49.

\section{Figures}

Fig. 1

a

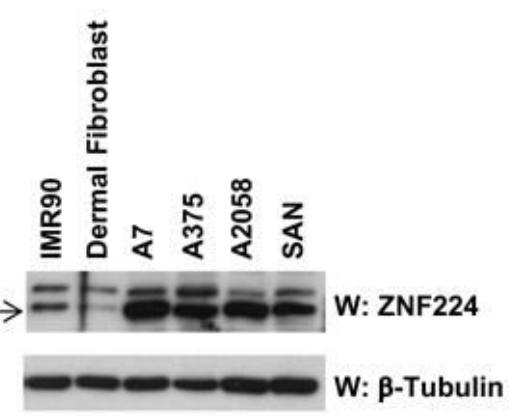

c

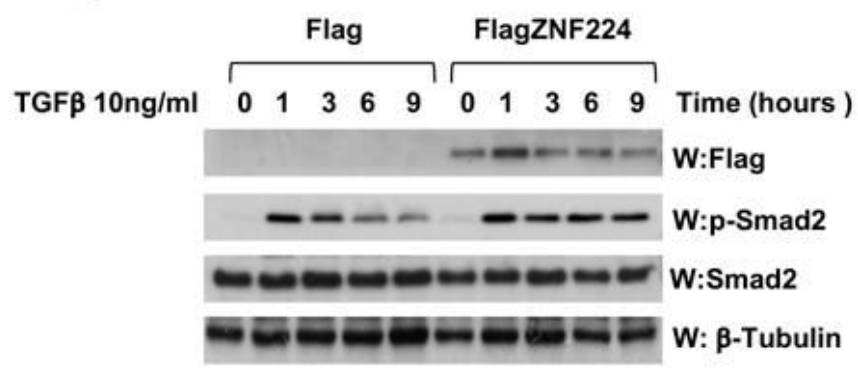

b
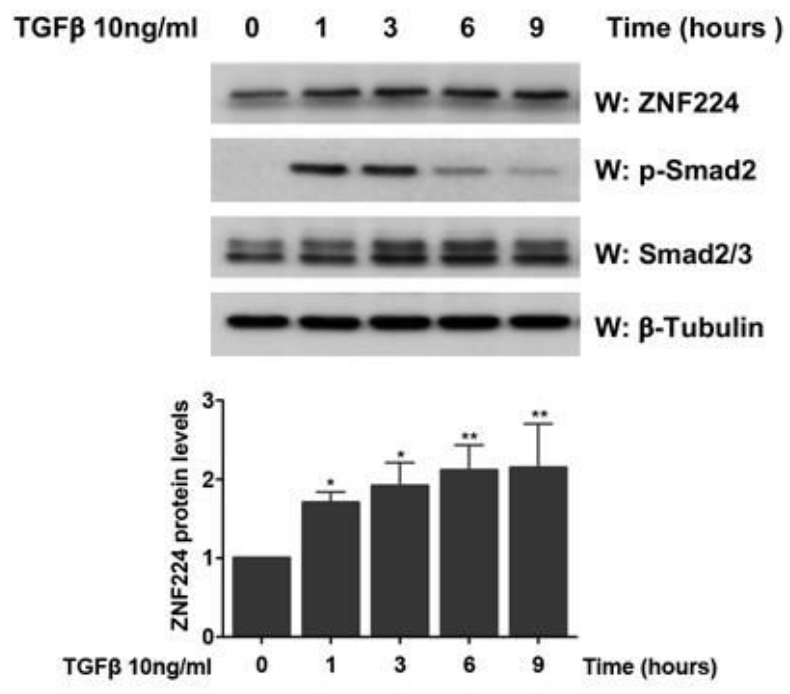

\section{Figure 1}

ZNF224 expression was induced by TGF- $\beta$ treatment and its overexpression prolonged Smad2/3 phosphorylation. a Western blot analysis of ZNF224 in protein extracts from melanoma cell lines (A7, A375, A2058, and SAN), human fibroblast cell line (IMR90), and human primary dermal fibroblasts. $\beta$ tubulin was used as a loading control. b Western Blot analysis of ZNF224, p-Smad2, and total Smad2/3 levels in A375 cells treated with TGF- $\beta$ for $1,3,6$, or 9 h. $\beta$-tubulin was used as a loading control. Densitometric analysis of ZNF224 protein levels is shown. $c$ Western Blot analysis of phosphorylated Smad2 and total Smad2 levels in A375 cells transfected with Flag-ZNF224 plasmid or its empty vector as a control and treated with TGF- $\beta 1$ for $1,3,6$, or 9 h. $\beta$-tubulin was used as a loading control. 
Fig.2

a

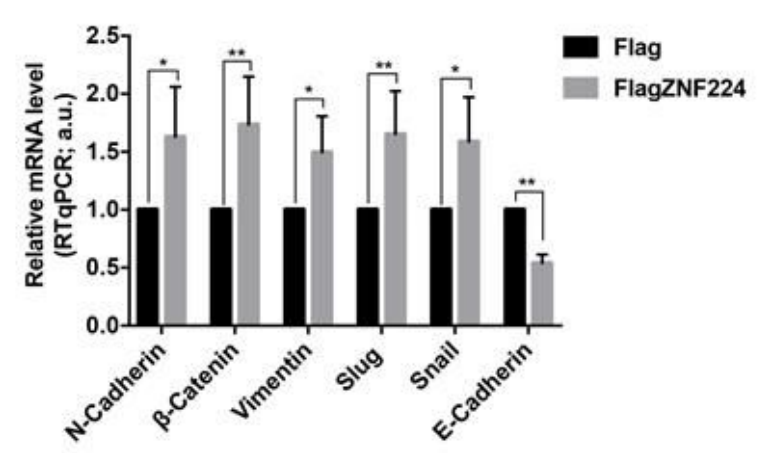

c

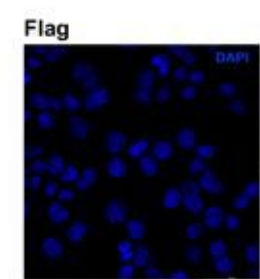

FlagZNF224

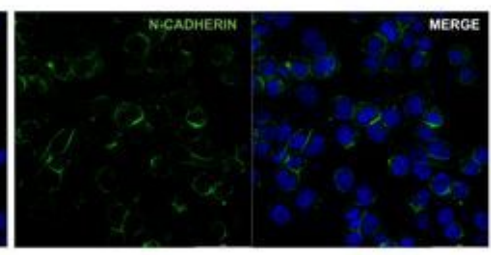

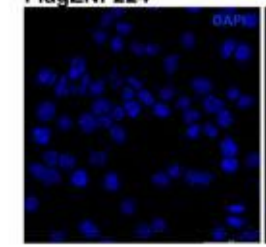

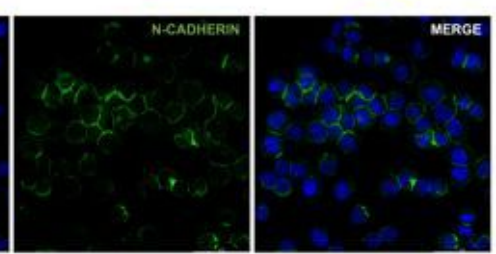

b

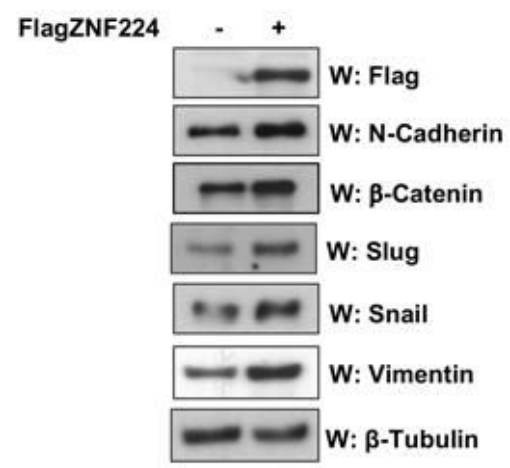

d

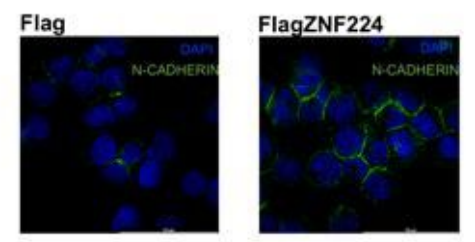

\section{Figure 2}

ZNF224 affected the expression of TGF- $\beta$ target genes. a RT-qPCR analysis of N-cadherin, $\beta$-catenin, Vimentin, Slug, Snail, and E-cadherin mRNA expression levels in A375 cells transfected with the FlagZNF224 plasmid or its empty vector (Flag). b Western Blot analysis of N-cadherin, $\beta$-catenin, Slug, Snail, and Vimentin expression levels in A375 cells transfected with the Flag-ZNF224 plasmid or its empty vector as a control. $\beta$-tubulin was used as a loading control. c N-cadherin immunofluorescence in A375 cells transfected with the Flag-ZNF224 construct. Microscopy images (Z-slices) showing N-cadherin expression in A375 cells overexpressing empty vector Flag (upper panel) and Flag-ZNF224 constructs (lower panel). d Regions of interest (ROls 1024x1024) showing a group of cells with a pronounced expression of N-cadherin in cells transfected with Flag-ZNF224 vector compared to the empty vector. Scale bar $=50 \mu \mathrm{m}$. 
b

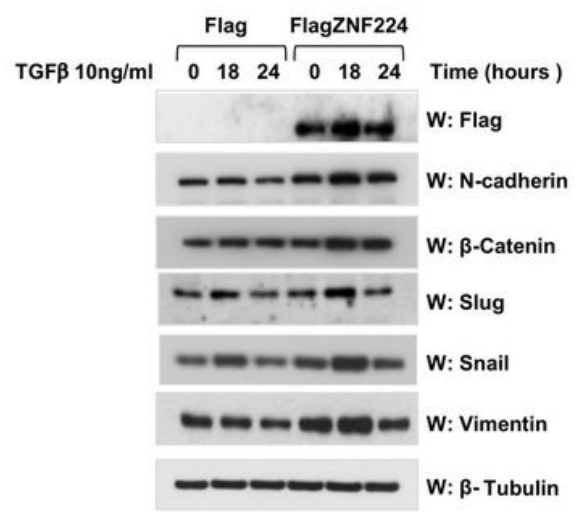

c

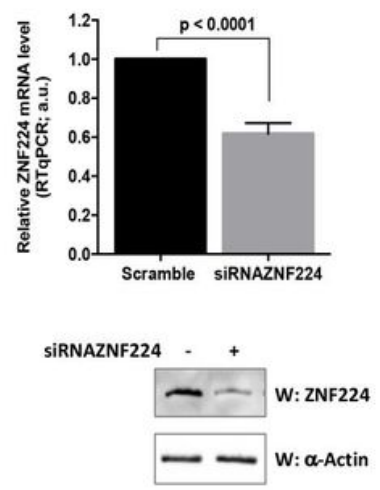

d

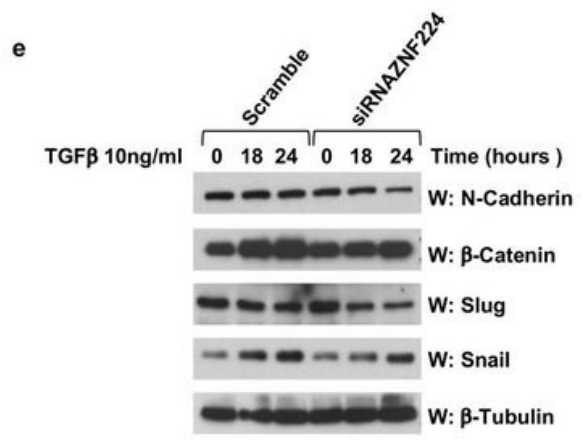

Figure 3

ZNF224 enhanced induction of TGF- $\beta$ responsive genes. a RT-qPCR analysis of N-cadherin, $\beta$-catenin, Snail, and Slug mRNA expression levels in A375 cells transfected with the Flag-ZNF224 plasmid or its empty vector as a control and stimulated with TGF- $\beta$ for 18 or $24 \mathrm{~h}$. b Western blot analysis of N-cadherin, $\beta$-catenin, Slug, Snail, and Vimentin protein levels in A375 cells transfected with the Flag-ZNF224 plasmid or its empty vector as a control and stimulated with TGF- $\beta$ for 18 or $24 \mathrm{~h}$. $\beta$-tubulin was used as a loading control. c RT-qPCR analysis of ZNF224 mRNA expression levels in A375 cells transfected with ZNF224 siRNA or scramble siRNA (upper panel). Western blot analysis of ZNF224 levels in A375 cells transfected with ZNF224 siRNA or scramble siRNA. a-actin was used as a loading control (lower panel). d RT-qPCR analysis of $\mathrm{N}$-cadherin, $\beta$-catenin, Vimentin, Slug, and Snail mRNA expression levels in A375 cells transfected with ZNF224 siRNA or scramble siRNA. e Western blot analysis of N-cadherin, $\beta$-catenin, Slug, and Snail protein levels in A375 cells transfected with ZNF224 siRNA or scramble siRNA and stimulated with TGF- $\beta$ for 18 or 24 h. $\beta$-tubulin was used as a loading control. 
Fig.4

a
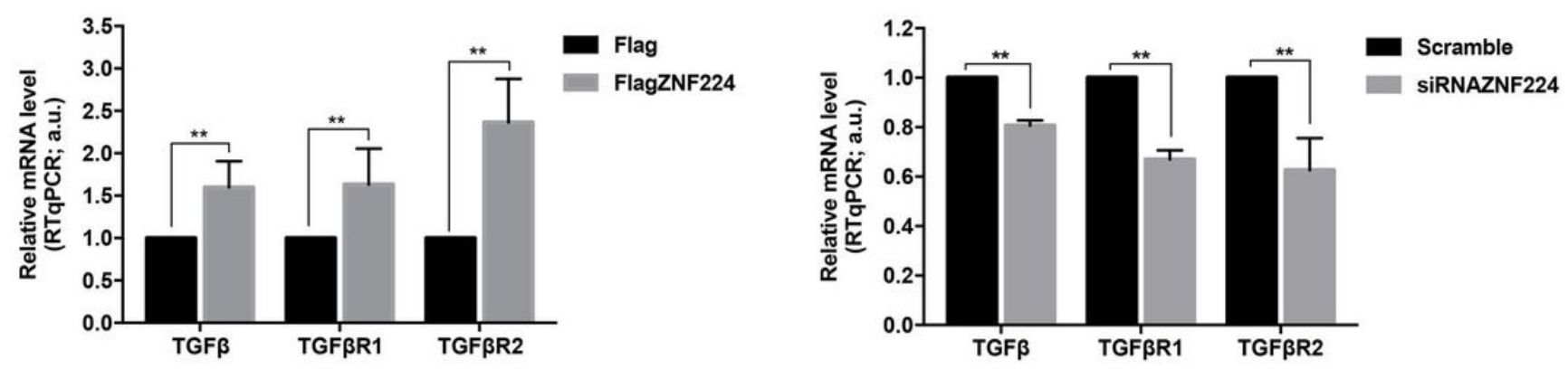

b
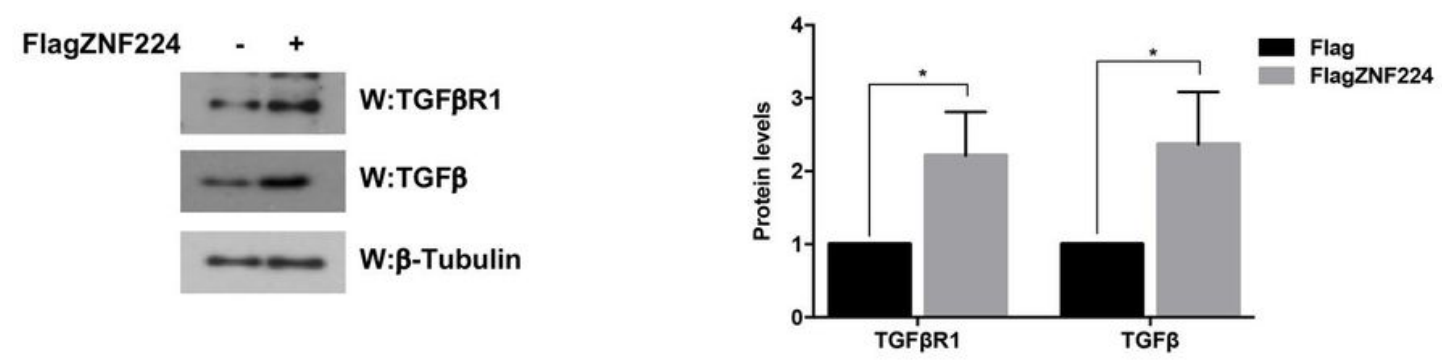

c
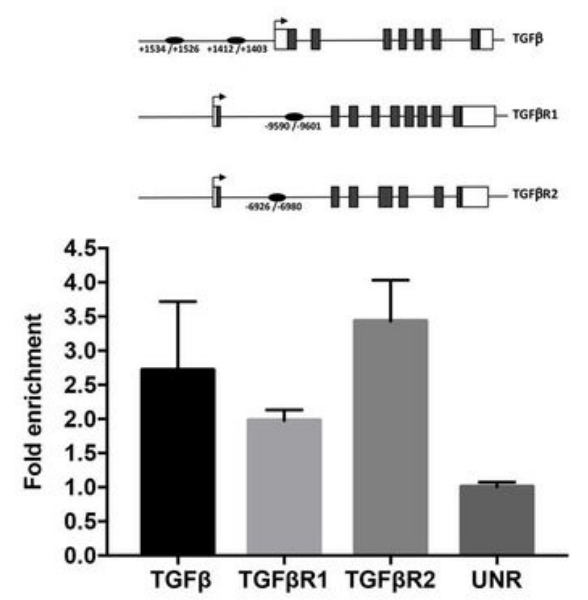

d

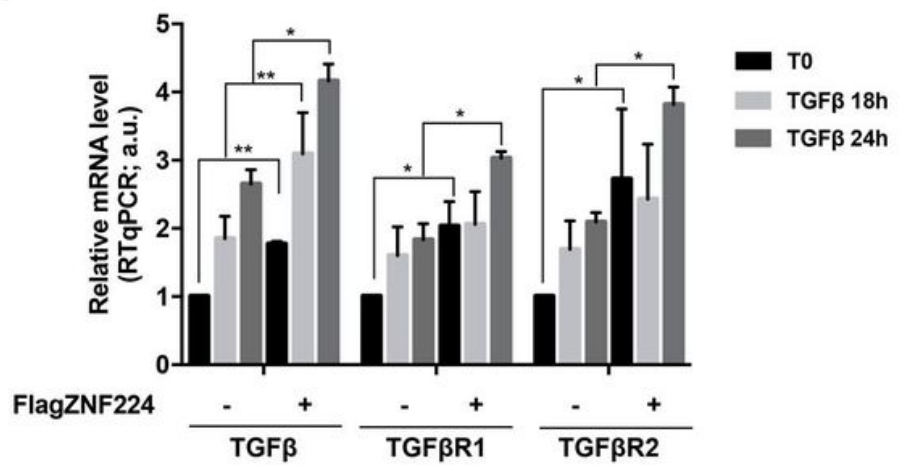

Figure 4

ZNF224 affected the expression of TGF- $\beta$, TGF $\beta R 1$, and TGF $\beta$ R2. a RT-qPCR analysis of TGF $\beta$, TGF $\beta R 1$, and TGF $\beta$ R2 mRNA levels in A375 cells overexpressing FlagZNF224 compared to cells transfected with the empty vector (Flag) (left panel) and in A375 cells transfected with ZNF224 siRNA or scramble siRNA (right panel). b Western blot analysis of TGF $\beta R 1$ and TGF $\beta$ protein levels in A375 cells transfected with the FlagZNF224 plasmid or empty vector (-). $\beta$-tubulin was used as a loading control. Densitometric analysis of TGF $\beta R 1$ and TGF $\beta$ protein levels is shown (right panel). c ZNF224 binds in vivo TGF $\beta$, TGFßR1, and TGFßR2 genes. X-Chlp assay was performed in A375 cells overexpressing Flag-ZNF224 with the Flag antibody or IgG as a control. The immunoprecipitated chromatin was analysed by RT-qPCR 
using specific primers spanning the putative ZNF224 binding sites shown in the upper panel. An unrelated region (UNR) was used as a negative control. d RT-qPCR analysis of TGF- $\beta$, TGF $\beta$ R1, and TGF $\beta R 1$ mRNA expression levels in A375 cells transfected with the Flag-ZNF224 plasmid or its empty vector as a control and stimulated with TGF- $\beta$ for 18 or $24 \mathrm{~h}$.

Fig. 5

a $\mathbf{3 7 5}$
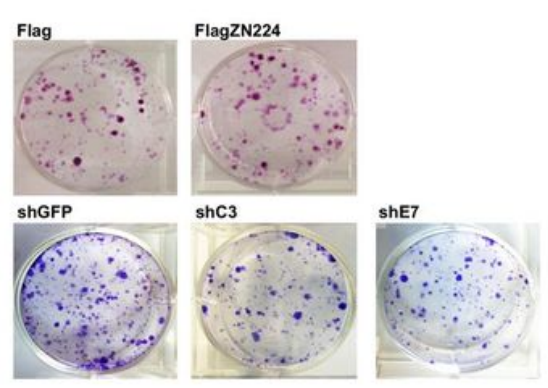

b
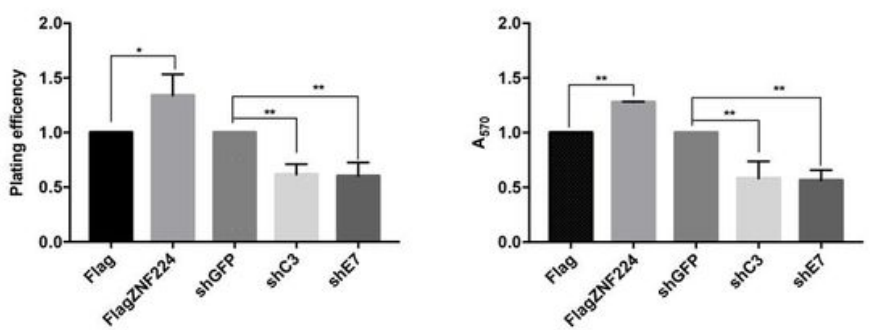

c

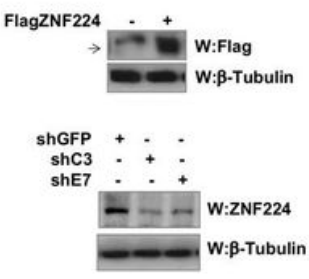

d 42058

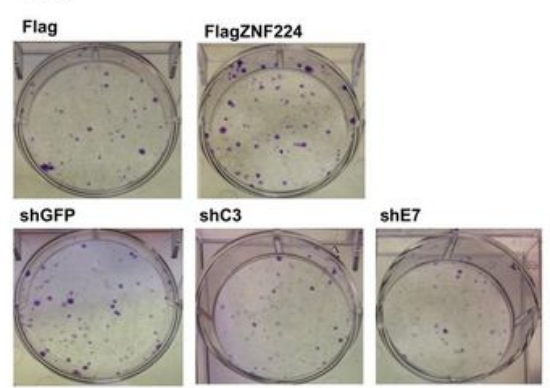

e
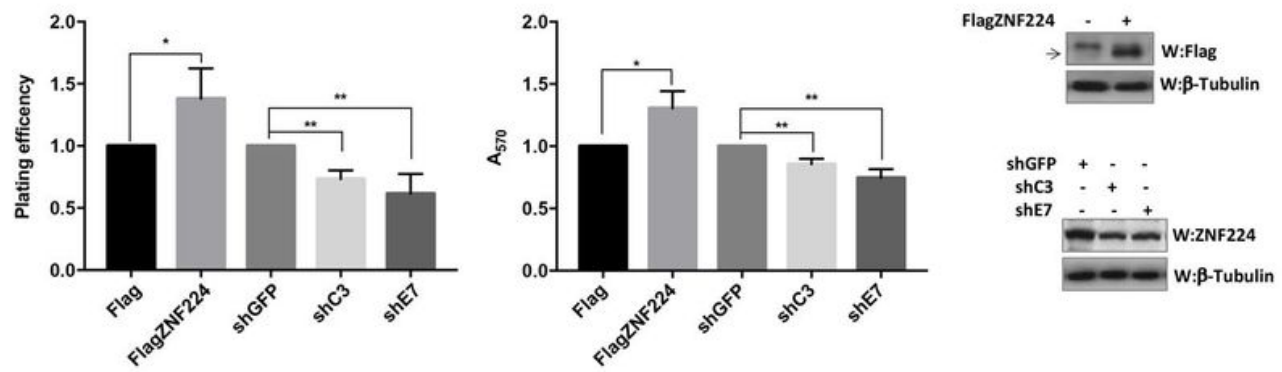

Figure 5

Effects of ZNF224 overexpression and silencing on A375 and A2058 cell growth. a Colony formation assay in A375 cells overexpressing (Flag-ZNF224) or silenced (shC3, shE7) for ZNF224 and their respective control cells (Flag and shGFP). b Plating efficiency and A570 after crystal violet elution were evaluated. Flag and shGFP control cells were arbitrarily set at 1 . The histograms represent the mean of two independent experiments performed in triplicate. c Western blot analysis was used to verify FlagZNF224 overexpression and ZNF224 silencing in A375 cells. $\beta$-tubulin was used as a loading control. $d$ Colony formation assay in A2058 cells overexpressing (Flag-ZNF224) or silenced (shC3, shE7) for ZNF224 and their respective control cells (Flag and shGFP). e Plating efficiency and A570 after crystal violet elution were measured. Flag and shGFP control cells were arbitrarily set at 1 . $f$ Western blot analysis was used to verify Flag-ZNF224 overexpression and ZNF224 silencing in A2058 cells. $\beta$-tubulin was used as a loading control. 
Fig.6

a

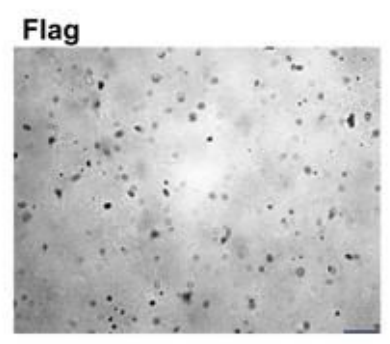

FlagZNF224

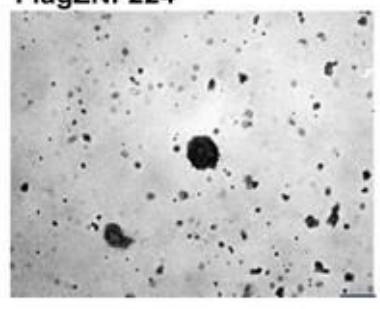

b

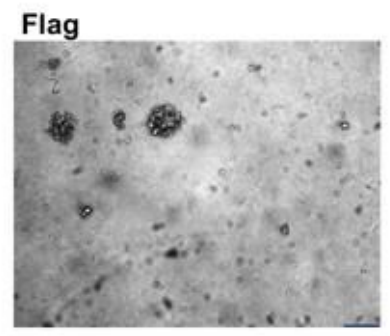

FlagZNF224

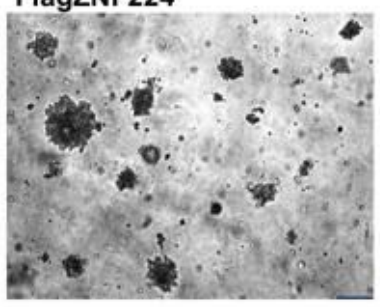

A375 cells

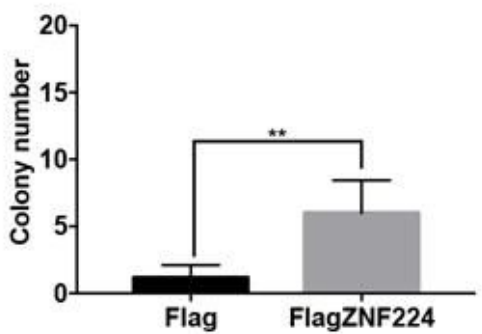

A2058

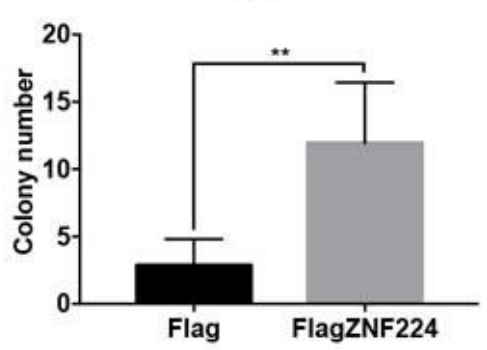

\section{Figure 6}

ZNF224 overexpression stimulated anchorage-independent growth of A375 and A2058 cells. Soft agar colony formation assay in A375 (panel a) and A2058 (panel b) cells overexpressing ZNF224 (FlagZNF224) compared to control cells (Flag). Twenty-four hours post-transfection, the cells were cultured in soft agar medium for two weeks. Then, the colonies were counted, and images of A375 and A2058 colonies were acquired using a light microscope. Scale bar $250 \mu \mathrm{m}$. The experiment was performed once in triplicate. 
${ }_{\text {Fig. }}$ a

A375

ZNF224 overexpression

Migration Assay

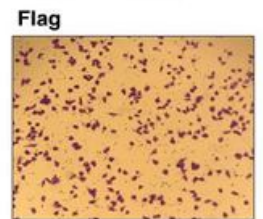

Invasion Assay

Flag
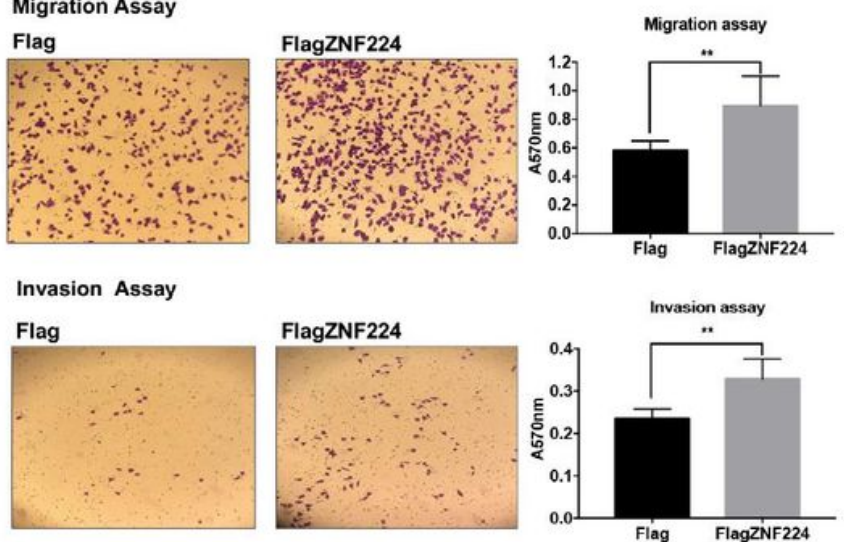

b

ZNF224 silencing

Migration Assay

Scramble
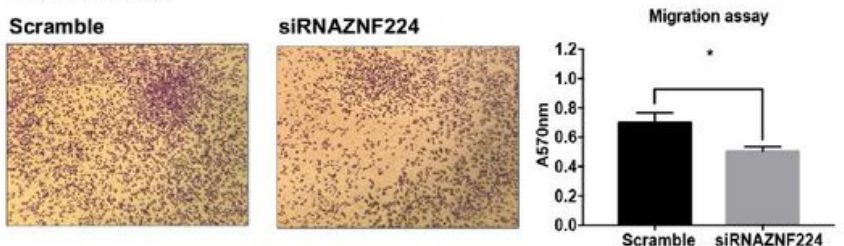

Invasion Assay

Scramble
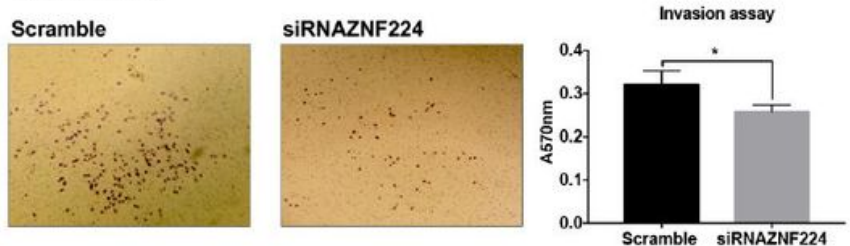
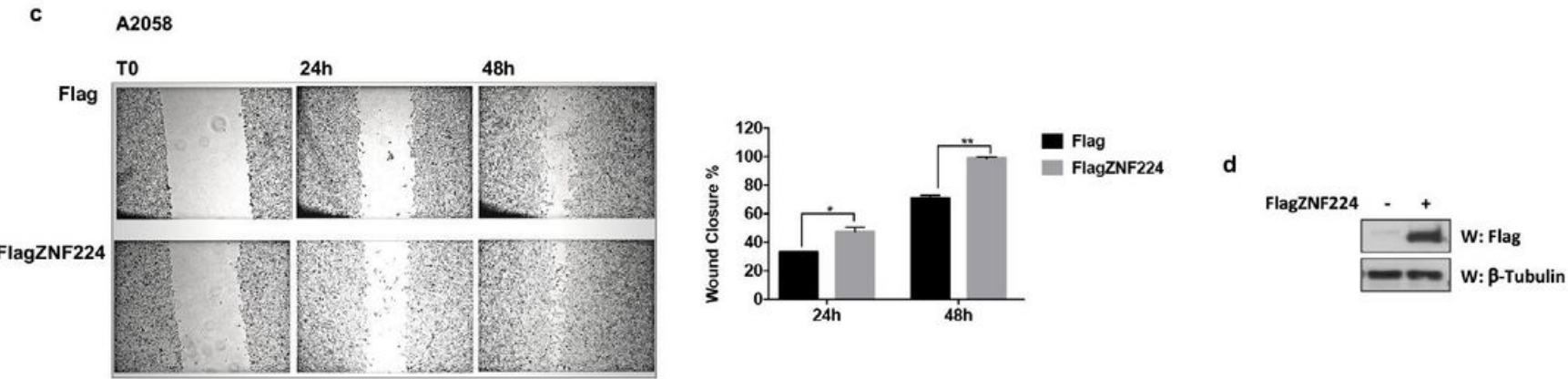

\section{Figure 7}

ZNF224 affected the migration and invasion of melanoma cells. a Representative images of the migration and invasion assay performed in A375 cells overexpressing ZNF224 (FlagZNF224) and control cells (Flag). b Representative images of the migration and invasion assay performed in A375 cells silenced for ZNF224 (siRNAZNF224) and control cells (scramble). The histograms of absorbance measured at $570 \mathrm{~nm}$ of eluted crystal violet were obtained from the mean of two independent experiments performed in triplicate. c A2058 cells overexpressing Flag-ZNF224 and control cells (Flag) were subjected to an in vitro scratch assay and the images were captured at 0,24 , and $48 \mathrm{~h}$ after the injury using a phase-contrast microscope. The histogram indicates the percentage of wound closure in the area. The expression of exogenous 3xFlag-ZNF224 protein in transfected cells was verified by western blot analysis. $\beta$-tubulin was used as a loading control (right panel). 